\title{
Hydromagnetic waves in a compressed-dipole field via field-aligned Klein-Gordon equations
}

\author{
Jinlei Zheng ${ }^{1}$, Qiang Hu ${ }^{1,2}$, Gary M. Webb ${ }^{2}$, and James F. McKenzie ${ }^{2,3, \dagger}$ \\ ${ }^{1}$ Department of Space Science, University of Alabama, Huntsville, AL, USA \\ ${ }^{2}$ Center for Space Plasma and Aeronomic Research (CSPAR), University of Alabama, Huntsville, AL, USA \\ ${ }^{3}$ Department of Mathematics and Statistics, Durban University of Technology, Steve Biko Campus, \\ Durban, South Africa \\ $\dagger$ deceased \\ Correspondence to: Qiang Hu (qh0001@uah.edu)
}

Received: 1 November 2015 - Revised: 5 April 2016 - Accepted: 19 April 2016 - Published: 2 May 2016

\begin{abstract}
Hydromagnetic waves, especially those of frequencies in the range of a few millihertz to a few hertz observed in the Earth's magnetosphere, are categorized as ultra low-frequency (ULF) waves or pulsations. They have been extensively studied due to their importance in the interaction with radiation belt particles and in probing the structures of the magnetosphere. We developed an approach to examining the toroidal standing Aflvén waves in a background magnetic field by recasting the wave equation into a Klein-Gordon (KG) form along individual field lines. The eigenvalue solutions to the system are characteristic of a propagation type when the corresponding eigenfrequency is greater than a critical frequency and a decaying type otherwise. We apply the approach to a compressed-dipole magnetic field model of the inner magnetosphere and obtain the spatial profiles of relevant parameters and the spatial wave forms of harmonic oscillations. We further extend the approach to poloidalmode standing Alfvén waves along field lines. In particular, we present a quantitative comparison with a recent spacecraft observation of a poloidal standing Alfvén wave in the Earth's magnetosphere. Our analysis based on the KG equation yields consistent results which agree with the spacecraft measurements of the wave period and the amplitude ratio between the magnetic field and electric field perturbations.
\end{abstract}

Keywords. Electromagnetics (wave propagation) - magnetospheric physics (magnetospheric configuration and dynamics) - space plasma physics (experimental and mathematical techniques)

\section{Introduction}

Hydromagnetic waves are common phenomena in space plasmas. The associated magnetic and electric field perturbations are observed both on the ground and from space in the Earth's magnetosphere. Such waves or magnetic pulsations of frequencies less than $\sim 1 \mathrm{~Hz}$ are typically categorized as ultra low-frequency (ULF) waves (Fraser, 2006; Kivelson, 2006). They can be further divided into subcategories, such as Pc1-5, Pi1-3 and Pg, with frequencies ranging from a few hertz down to a few millihertz (Fraser, 2006; Volwerk, 2006). Sometimes they exhibit regular and monochromatic magnetic and electric field wave forms which are due to the standing wave mode along magnetic field lines. Such waves can be identified as Alfvén waves propagating in the Earth's magnetosphere, e.g., the recent spacecraft observation by the Van Allen Probes (Radiation Belt Storm Probes) of Dai et al. (2013). Based on the direction or the polarization of the magnetic (or electric) field perturbation in the linearized assumption, they can be further characterized as toroidal and/or poloidal-mode waves. In the toroidal mode, the magnetic field perturbation is in the azimuthal direction, i.e., along the east-west longitudinal direction (the accompanying electric field perturbation has a radial component) in Earth's dipole magnetic field. On the other hand, in the poloidal mode, the magnetic field perturbation has a radial component, lying in the meridional plane, while the electric field perturbation is azimuthal.

The basic theory for ULF waves can be traced back to Dungey $(1955,1963)$ and Tamao (1965) and has been well 
developed. These waves are interpreted as standing Alfvén (transverse) or fast-mode hydromagnetic waves in cold plasmas immersed in the Earth's magnetic field (Cummings et al., 1969; Singer et al., 1981; Southwood and Hughes, 1983). Their characteristics are closely governed by the geometry of the background magnetic field and the associated plasma density distribution. More general and sophisticated numerical simulations were also developed in recent years to take into account more realistic background field topology, multiple physical effects and non-idealized boundary conditions (Kabin et al., 2007; Lee and Takahashi, 2006; Claudepierre et al., 2010; Degeling et al., 2010). The study of ULF waves has important implications for wave-particle interaction and diagnostics of magnetospheric structures. In particular, the critical role that ULF waves play in the energization and transport of radiation belt particles has been established based on both theoretical and observational studies (Elkington, 2006; Elkington et al., 2003, 1999; Takahashi et al., 2002; Ukhorskiy et al., 2005; Dimitrakoudis et al., 2015).

An alternative approach to describing the toroidal (transverse) Alfvén standing waves in an axisymmetric background magnetic field has been given by McKenzie and $\mathrm{Hu}$ (2010), where the wave equations were cast along an individual field line and transformed into a Klein-Gordon (KG) form. This approach was further formalized and applied to the Earth's dipole magnetic field. We later showed in great detail the formulation and procedures of the approach for a given background field topology and density distribution in Webb et al. (2012). The eigenfrequencies obtained from the eigenmode solutions to the KG equations correspond well to the ULF waves frequencies in the Pc3-5 range, from a few to tens of millihertz (Webb et al., 2012). The same approach was also successfully applied to coronal loop oscillations in low corona under different background field and plasma conditions (Hu et al., 2012). In the present work, we first apply the approach to a more realistic Earth background field as represented by a compressed-dipole model (e.g., Kabin et al., 2007). We derive the eigenfrequencies and eigenfunctions of the wave forms for this particular geometry corresponding to the toroidal mode and compare the results with other similar studies.

Furthermore, motivated by a recent direct observation of poloidal standing Alfvén waves in Earth's magnetosphere by Dai et al. (2013) (see also Dai et al., 2015; Takahashi et al., 2013; Liu et al., 2013, 2011), we extend our investigation to examine the poloidal-mode waves as well. In the case of a transverse poloidal mode, we show that the wave equation can also be cast as a KG form along a field line. We then numerically solve the wave equation for electric field perturbation. The corresponding magnetic field perturbation can be obtained in a similar manner to the approach based on KG equations for the toroidal mode. In Dai et al. (2013), an event of a fundamental-mode standing poloidal wave was identified from the Van Allen Probes measurements. They obtained the wave period of the azimuthal electric field and the asso- ciated radial magnetic field oscillations, the relative ratio of wave amplitudes, and the relative phase shift at the spacecraft location in the inner magnetosphere. Their analysis provided direct evidence for the existence of poloidal-mode waves and their interaction with particles. In addition, the quantitative measurements of wave properties can be directly compared with our model output.

This article is organized as follows. Section 2 provides a brief summary of the toroidal-mode wave equations and their transformation into the KG form. The general approach of solving the resulting eigenvalue problem is described and applied to a compressed-dipole magnetic field model of Earth's magnetosphere. The eigenfrequencies and the corresponding wave-form solutions are presented. Section 3 extends the analysis to the decoupled eigenvalue solutions of the poloidal-mode waves for a given geometry and presents a quantitative comparison of the standing transverse Alfvén wave solutions with the observations of Dai et al. (2013). Finally, we summarize our results in the last section.

\section{Klein-Gordon equations for the toroidal mode}

We first consider toroidal wave perturbations $\left(b_{\phi}, u_{\phi}\right)$ in the magnetic field and fluid velocity in a background axisymmetric (that is, azimuthal wave number $m=0$ ) magnetic field $\boldsymbol{B}_{0}=\left(B_{r}, B_{\theta}, 0\right)$, in spherical coordinates $(r, \theta, \phi)$. The perturbation electric field $\boldsymbol{E}$ is given by

$\boldsymbol{E}=-\boldsymbol{u} \times \boldsymbol{B}=-u_{\phi} B_{r} \hat{\theta}+u_{\phi} B_{\theta} \hat{r}=E_{\mathrm{n}} \hat{n}$,

normal to the background magnetic field line. The $\phi$ (toroidal) components of Faraday's law and the momentum equation yield the following wave equations for the perturbations, when evaluated along individual field lines that can be specified by a functional form $r(\theta)$ between the radial distance $r$ and the colatitude $\theta$ (McKenzie and Hu, 2010; Webb et al., 2012):

$$
\begin{aligned}
\frac{\partial^{2} b_{\phi}}{\partial t^{2}} & =\frac{V^{2}}{r^{2}}\left\{\frac{\mathrm{d}^{2} b_{\phi}}{\mathrm{d} \theta^{2}}-\frac{1}{L_{b}} \frac{\mathrm{d} b_{\phi}}{\mathrm{d} \theta}+\frac{b_{\phi}}{M_{b}}\right\} \\
\frac{\partial^{2} u_{\phi}}{\partial t^{2}} & =\frac{V^{2}}{r^{2}}\left\{\frac{\mathrm{d}^{2} u_{\phi}}{\mathrm{d} \theta^{2}}-\frac{1}{L_{u}} \frac{\mathrm{d} u_{\phi}}{\mathrm{d} \theta}+\frac{u_{\phi}}{M_{u}}\right\},
\end{aligned}
$$

where $V=B_{\theta} / \sqrt{\mu_{0} \rho}$ with a given background plasma density $\rho$ and all coefficients, $\frac{1}{L}$ and $\frac{1}{M}$, are functions of $\theta$ only. The particular forms of these coefficients for the velocity perturbation, Eq. (3), are (Webb et al., 2012)

$$
\begin{aligned}
& -\frac{1}{L_{u}}=\frac{\mathrm{d}}{\mathrm{d} \theta} \ln \left(\frac{B_{\theta}}{r}\right), \quad \frac{1}{M_{u}}=-\frac{r}{B_{\theta}} \frac{\mathrm{d}}{\mathrm{d} \theta}\left(\frac{B_{\theta}}{r l_{u}}\right)-\frac{1}{l_{u}^{2}}, \\
& \frac{1}{l_{u}}=\cot \theta+\frac{B_{r}}{B_{\theta}} .
\end{aligned}
$$

The total differentiation along a field line is given

$$
\frac{\mathrm{d}}{\mathrm{d} \theta}=\frac{\partial}{\partial \theta}+\frac{r B_{r}}{B_{\theta}} \frac{\partial}{\partial r} \text {. }
$$


These wave equations are to be solved along individual field lines by being transformed into (linear) Klein-Gordon equations of the ordinary differential equation type. The solutions are obtained for a given background magnetic field topology and the associated density distribution and harmonic time dependence, subject to specific boundary conditions. The detailed derivation, formulations and procedures are given in Webb et al. (2012), including a case study of a standarddipole field. We restrict our presentation mostly to a brief description of the general case below.

\subsection{General case}

The perturbations of physical quantities as given by Eqs. (2) and (3) have the general form

$$
\frac{\partial^{2} \Psi}{\partial t^{2}}=\frac{V^{2}}{r^{2}}\left[\frac{\mathrm{d}^{2} \Psi}{\mathrm{d} \theta^{2}}-\frac{1}{\mathcal{L}} \frac{\mathrm{d} \Psi}{\mathrm{d} \theta}+\frac{1}{\mathcal{M}} \Psi\right]
$$

which can be transformed into the Klein-Gordon form through the substitution

$$
\Psi=\psi \exp \int \frac{\mathrm{d} \theta}{2 \mathcal{L}} \text {. }
$$

This yields the KG equation (Morse and Feshbach, 1953)

$$
\frac{\partial^{2} \psi}{\partial t^{2}}+\omega_{c}^{2} \psi=\frac{V^{2}}{r^{2}} \frac{\mathrm{d}^{2} \psi}{\mathrm{d} \theta^{2}}
$$

in which a critical frequency $\omega_{c}$ is manifest and given by

$\omega_{c}^{2}=\frac{V^{2}}{r^{2}}\left[\frac{1}{2 \mathcal{L}^{2}}\left(1+\mathcal{L}^{\prime}\right)-\frac{1}{\mathcal{M}}\right]$.

The amplitude factor in Eq. (7) is defined as

$\exp \int \frac{\mathrm{d} \theta}{2 \mathcal{L}}=\frac{1}{\sqrt{F(\theta)}}=f(\theta)$.

This factor arises from the adiabatic geometric growth or decay corresponding to the conservation of wave energy flux through a flux tube as given by Poynting's theorem (McKenzie and $\mathrm{Hu}, 2010)$. For the velocity perturbation, in particular, the relevant factor is simply $f(\theta)=\left(B_{\theta} / r\right)^{-\frac{1}{2}}$ (Webb et al., 2012). That the quantity $\omega_{c}$, given by (9), in Eq. (8) is indeed a critical frequency as is readily seen by taking a harmonic time variation $\propto \exp (i \omega t)$, for then Eq. (8) becomes

$$
\frac{\mathrm{d}^{2} \psi}{r^{2} \mathrm{~d} \theta^{2}}=-\frac{\left(\omega^{2}-\omega_{c}^{2}\right)}{V^{2}} \psi \equiv-k^{2} \psi .
$$

An equation of this form possesses propagating-type solutions, provided $\omega>\omega_{c}$ (or $\omega^{2}>\omega_{c}^{2}$ ), and decaying solutions for $\omega<\omega_{c}$. If a slowly varying background is assumed, Jeffreys-Wentzel-Kramers-Brillouin (JWKB) solutions yield good approximations to the propagating and nonpropagating behavior. The imposition of boundary conditions (e.g., at the end points of one field line) yield an eigenvalue problem for $k$ (and hence $\omega$ ). Furthermore, by a change of variable (Webb et al., 2012), $r d \theta=\frac{B_{\theta}}{B} d s$, where the fieldline segment is denoted by $d s$, the above equation can be written $\left(V_{A}=B / \sqrt{\mu_{0} \rho}\right.$, the Alfvén speed) as

$$
\frac{\mathrm{d}^{2} \psi}{\mathrm{d} s^{2}}=-\frac{\left(\omega^{2}-\omega_{c}^{2}\right)}{V_{A}^{2}} \psi
$$

A normal-mode analysis (plane wave approximation) yields a dispersion relation $\omega^{2}=\omega_{c}^{2}+k^{2} V_{A}^{2}$. So the propagating mode only exists for $\omega$ exceeding $\omega_{c}$. Such a threshold does not exist, however, for $\omega_{c}^{2}<0$.

An important and general treatment of the wave modes and their coupling was developed by Chen and Cowley (1989, and references therein), especially in the context of fieldline resonances. We share the same theoretical basis in that we start with ideal magnetohydrodynamic (MHD) equations and arrive at the equations describing the electric field perturbations. In particular, those authors derived the eigenfunction equation (Eq. 11 therein) for the toroidal-mode standing Alfvén waves in a dipole field. The eigenfrequencies are real and the corresponding eigenfunctions form a complete and orthogonal set. Its field-aligned form is similar to Eq. (12) above, although without the explicit critical frequency embedded. In our study, we explicitly assume axisymmetry (corresponding to azimuthal wave number $m=$ 0 ), by which the different wave modes are decoupled. We then seek regular solutions of eigenfunctions corresponding to discrete real eigenfrequencies along individual field lines for a given background field geometry that goes beyond a standard-dipole field. So our approach and results are more directly comparable with those of Cummings et al. (1969).

It is also worth noting that the Green's functions for the KG equation are well known (Morse and Feshbach, 1953), although for the cases of constant coefficients. They involve parameters characterizing the system and the surrounding medium in an infinite domain. The characteristics of the Green's functions are reflected in our solutions. For example, the Green's function for an infinite domain also shows "a characteristically 'damped' space dependence" (Morse and Feshbach, 1953) in a limit similar to the one discussed above. The Green's function approach is especially advantageous in dealing with time-dependent boundary conditions. However, in our present formulation, it is not clear how a closed-form Green's function can be obtained for the KG equation of spatially varying coefficients. Therefore, in the present study, we focus on a limited scope in seeking numerical eigenvalue solutions to the $\mathrm{KG}$ equation subject to a set of homogeneous boundary conditions within a finite spatial domain in order to carry out a comparison with direct spacecraft observations in Earth's magnetosphere.

The procedures for solving the toroidal wave equations were given by Webb et al. (2012) and Hu et al. (2012). We adopt the usual boundary condition $E_{\mathrm{n}} \equiv 0$ (i.e., $u_{\phi}=0$ ) when solving the eigenvalue problem for $u_{\phi}$ satisfying the KG Eq. (11). Physically, this corresponds to the situation 
of the field-line footpoints rooted in the Earth's ionosphere of infinite conductivity. The toroidal velocity perturbations are then obtained by solving the KG equation subject to the boundary condition and the transformation of the amplitude factor. A set of solutions of different wave forms is obtained for a discrete set of eigenvalues $\omega$, which usually correspond to a set of harmonic oscillations with increasing frequency and number of nodes (Webb et al., 2012). Then the accompanying toroidal magnetic field perturbation is calculated by

$$
\frac{\partial b_{\phi}}{\partial t}=\frac{B_{\theta}}{r} \frac{d u_{\phi}}{\mathrm{d} \theta}+\frac{u_{\phi} B_{\theta}}{r} \frac{1}{l_{b}},
$$

where the coefficient $\frac{1}{l_{b}}=-\frac{1}{l_{u}}$ is known once the background magnetic field topology is given. Depending on the specific eigenmode solution being sought, a constant eigenfrequency $\omega$ and the corresponding eigenfunction solutions are obtained for both $u_{\phi}$ and $b_{\phi}$.

As examples, the cases of a standard-dipole field with a typical power-law density distribution have been examined for ULF waves in Earth's magnetosphere (Webb et al., 2012) and coronal loop oscillations in the Sun's corona (Hu et al., 2012) by the above approach. Figure 1 shows the variation of the critical frequency $\omega_{c}$ and the amplitude factor $f(\theta)$ for an axisymmetric dipole field of the Earth, particularly for $L=2,4,6$ (here the value $L$, as in " $L$ shell", represents the radial distance of one particular field line crossing the Equator). We use a density model by Kabin et al. (2007) throughout the present study except for where noted: $\rho=\rho_{e}\left(\frac{5}{r}\right)^{4}$ with $\rho_{e}=7 \mathrm{amu} \mathrm{cm}{ }^{-3}$ and the radial distance $r$ is measured in Earth radius. The general profiles of $\omega_{c}$ and $f$ are similar to those presented in Webb et al. (2012), but their magnitudes are sensitive to the different background density distributions assumed, as are the eigenfrequencies obtained. Table 1 lists the eigenfrequency of the fundamental-mode $\omega_{0}$, the corresponding period $T_{0}$ and locations $\theta_{0}$ along each individual field line where $\omega_{0}=\omega_{c}\left(\theta_{0}\right)$ for the dipole field. Given the profiles of $\omega_{c}(\theta)$ in Fig. 1, we find that for $\theta_{0}<\theta<\pi-\theta_{0}$ where $\omega>\omega_{c}$, the solution of the propagation type exists, while beyond that interval where $\omega<\omega_{c}$, a decaying-type solution exists, as reflected in the resulting wave forms from the corresponding eigenfunction solutions (see Webb et al., 2012). Clearly in this case, for one particular $L$ shell, the waves of frequencies less than the minimum of the corresponding critical frequency $\omega_{c}$ will not exist; thus, the finite real-valued $\omega_{c}$ does provide a lower bound on the allowable wave frequencies. The same set of results obtained from the case of a compressed-dipole field is to be presented in the following subsection.

\subsection{A compressed-dipole field}

A compressed-dipole field is given in the spherical coordinate (which is intrinsically a 3-D field but remains planar at
Table 1. List of parameters for $L=2,4,6$ of a standard-dipole field.

\begin{tabular}{ccccc}
\hline$L$ & $\omega_{0}, \mathrm{~s}^{-1}$ & $T_{0}, \mathrm{~s}$ & $\theta_{0},{ }^{\circ}$ & $r_{0}\left(R_{\mathrm{E}}\right)$ \\
\hline 2 & 0.95 & 6.6 & 49 & 1.15 \\
4 & 0.20 & 32 & 52 & 2.48 \\
6 & 0.085 & 74 & 53 & 3.81 \\
\hline
\end{tabular}
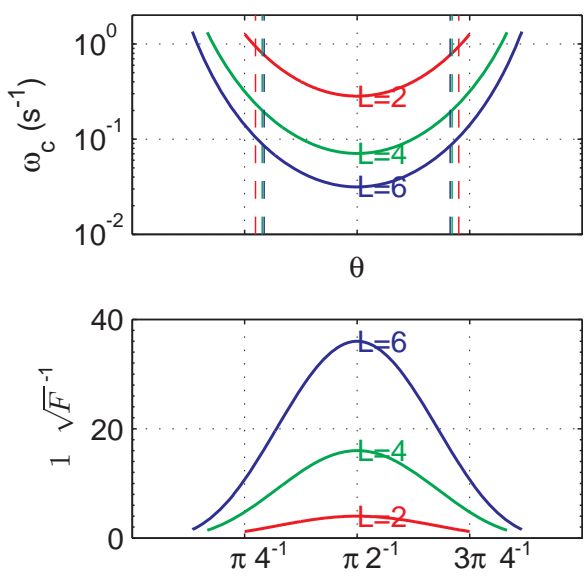

Figure 1. The parameter $\omega_{c}$ (with $B_{0}=0.31$ Gauss, $a=6.4 \times$ $10^{8} \mathrm{~cm}$ and $\rho_{e}=7 \mathrm{amu} \mathrm{cm}{ }^{-3}$ ) and the amplitude factor as a function of $\theta$ for various $L$ values of a dipole field. The vertical lines mark the location (colatitude) where $\omega_{c}=\omega_{0}$, the eigenfrequency of the corresponding fundamental mode.

each $\phi$ ) (Kabin et al., 2007):

$$
\begin{aligned}
B_{r} & =\left(\frac{2 B_{0}}{r^{3}}-b_{1}\left(1+b_{2} \cos \phi\right)\right) \cos \theta \\
B_{\theta} & =\left(\frac{B_{0}}{r^{3}}+b_{1}\left(1+b_{2} \cos \phi\right)\right) \sin \theta \\
B_{\phi} & =0 .
\end{aligned}
$$

So our approach based on axisymmetric geometry and wave Eq. (6) can only be approximately applied to the noonmidnight meridional planes corresponding to $\phi=0$ and $\phi=$ $\pi$, respectively, on which $\partial / \partial \phi=0$.

Since the field remains planar (i.e., $B_{\phi}=0$ ), it is possible to derive the field-line equation in each meridional plane $(r$, $\theta, \phi \equiv$ Const) with $r$ normalized by the Earth radius:

$\frac{\mathrm{d} r}{\mathrm{~d} \theta}=\frac{r B_{r}}{B_{\theta}}=\frac{r\left(2 / r^{3}-D\right)}{1 / r^{3}+D} \cot \theta$,

where we define a dimensionless quantity $D \equiv \frac{b_{1}}{B_{0}}(1+$ $b_{2} \cos \phi$ ). This leads to

$H \sin ^{2} \theta=\frac{r}{D \cdot r^{3}-2}$,

where $H$ is an integration constant. As usual, if we define $r=$ $L$ when $\theta=\pi / 2\left(L\right.$ shell), we obtain $H=L /\left(D \cdot L^{3}-2\right)$. 


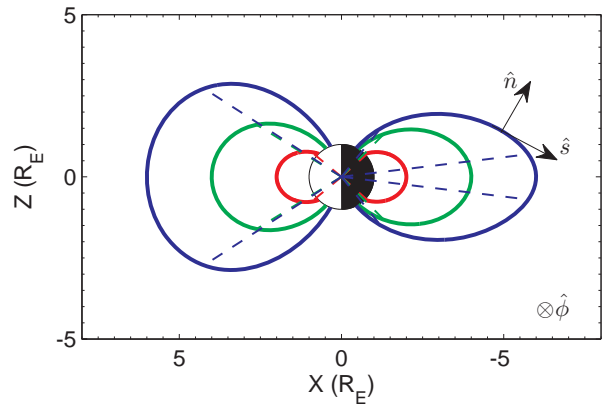

Figure 2. The magnetic field lines of $L=2,4,6$ on the noonmidnight meridional plane for the compressed dipole with $B_{0}=$ 0.31 Gauss, $b_{1}=10 \mathrm{nT}$ and $b_{2}=8$. The dashed lines mark the locations where the eigenfrequencies of the fundamental mode intersect the critical frequencies, i.e., $\omega_{0}=\omega_{c}$ as shown in Fig. 3 and given in Tables 2 and 3. The field-line-aligned orthogonal coordinate $(n, s, \phi)$ is also shown.

Table 2. List of parameters for $L=2,4,6$ of a compressed-dipole field for $\phi=0$ (noon).

\begin{tabular}{ccccc}
\hline$L$ & $\omega_{0}, \mathrm{~s}^{-1}$ & $T_{0}, \mathrm{~s}$ & $\theta_{0},{ }^{\circ}$ & $r_{0}\left(R_{\mathrm{E}}\right)$ \\
\hline 2 & 0.96 & 6.6 & 50 & 1.20 \\
4 & 0.22 & 29 & 58 & 3.06 \\
6 & 0.10 & 60 & 57 & 5.05 \\
\hline
\end{tabular}

Then the colatitude $\theta_{F}$ of one of the footpoints of one particular $L$ shell is obtained by $\sin ^{2} \theta_{F}=1 /(H \cdot D-2 H)$.

Subsequently, the necessary coefficients and factor in the KG Eq. (11) for the velocity perturbation in the toroidal mode are obtained as follows:

$$
\begin{aligned}
\frac{1}{l_{u}} & =-\frac{1}{l_{b}}=\frac{3}{1+D \cdot r^{3}} \cot \theta, \\
\frac{1}{L_{u}} & =\frac{7-4 D \cdot r^{3}-2 D^{2} r^{6}}{\left(1+D \cdot r^{3}\right)^{2}} \cot \theta .
\end{aligned}
$$

Additional coefficients such as $1 / M_{u}$ can be derived from Eq. (4) (Webb et al., 2012). Then Eq. (9) enables the derivation of an analytic form of the critical frequency $\omega_{c}$ appearing in the $\mathrm{KG}$ equation and the amplitude factor $f(\theta)$, relating the solution of the $\mathrm{KG}$ equation to the original physical perturbation quantity.

Figure 2 shows the selected field lines for $L=2,4$ and 6 , respectively, in both the noon $(\phi=0)$ and midnight $(\phi=\pi)$ meridional planes of the Earth as illustrated. The asymmetry between the two sides is clearly seen due to the compression of the solar wind on the noon side (X $>0)$. We carry out the analysis of toroidal-mode waves for each individual field line via the approach of KG equations outlined in Sect. 2.1.

First of all, the profiles of the critical frequency $\omega_{c}$ and the amplitude factor are calculated and illustrated in Fig. 3, together with the locations where the eigenfrequencies of the fundamental mode intersect the critical frequencies. The
Table 3. List of parameters for $L=2,4,6$ of a compressed-dipole field for $\phi=\pi$ (midnight).

\begin{tabular}{ccccc}
\hline$L$ & $\omega_{0}, \mathrm{~s}^{-1}$ & $T_{0}, \mathrm{~s}$ & $\theta_{0},{ }^{\circ}$ & $r_{0}\left(R_{\mathrm{E}}\right)$ \\
\hline 2 & 0.94 & 6.7 & 48 & 1.11 \\
4 & 0.18 & 34 & 45 & 1.86 \\
6 & 0.060 & 105 & $83(39)$ & $5.81(1.90)$ \\
\hline
\end{tabular}
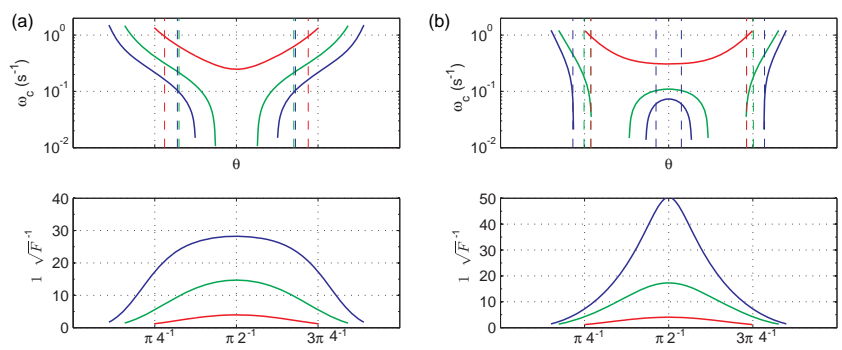

Figure 3. The parameter $\omega_{c}$ (with $B_{0}=0.31$ Gauss, $a=6.4 \times$ $10^{8} \mathrm{~cm}$ and $\rho_{e}=7 \mathrm{amu} \mathrm{cm}{ }^{-3}$ ) and the amplitude factor as a function of $\theta$ for various $L$ values for the compressed-dipole field with (a) $\phi=0$ and (b) $\phi=\pi$, respectively. Format is the same as Fig. 1 . The broken part of some curves corresponds to $\omega_{c}^{2}<0$.

corresponding parameters of the eigenfrequency $\omega_{0}$, the period $T_{0}$, the colatitude $\theta_{0}$ and the radial distance $r_{0}$ where $\omega_{c}=\omega_{0}$ are given in Tables 2 and 3 for the noon and midnight side, respectively. The profiles of $\omega_{c}$ and $f$ show significant differences among the cases of noon, midnight meridional plane of the compressed dipole, and that of a standard dipole, especially for greater $L$ values. For example, for the case $L=6$ on the midnight side, the amplitude factor peaks at a greater value $(\sim 50)$ at the Equator, while the eigenfrequency $\omega_{0}$ intersects the critical frequency at two locations in $\theta<\pi / 2$, one near the North Pole and the other near the Equator. Therefore, there are two separate regions of propagating solution to the KG equation where $\omega^{2}>\omega_{c}^{2}$ and one additional region of decaying solution surrounding the Equator as marked by the pairs of dashed blue lines in Fig. 3b along congruent points in colatitude. However, for higherorder harmonics, the eigenfrequency increases with the increasing number of nodes such that it becomes greater than the critical frequency throughout the whole range of low latitudes enclosing the Equator.

Overall, the values of parameters for the fundamental mode are comparable among the cases presented in Tables 13 , although significant deviations also exist, especially for the case of $L=6$. The periods range between a few to tens of seconds and a little over $100 \mathrm{~s}$, with increasing $L$ values, which correspond well to the frequency range of Pc1-5 ULF waves in Earth's magnetosphere. For the compresseddipole cases, the periods also agree well with those reported by Kabin et al. (2007), where the periods rose from a few seconds at $L=2$, to tens of seconds at $L=4$ and to $\sim 100$ 

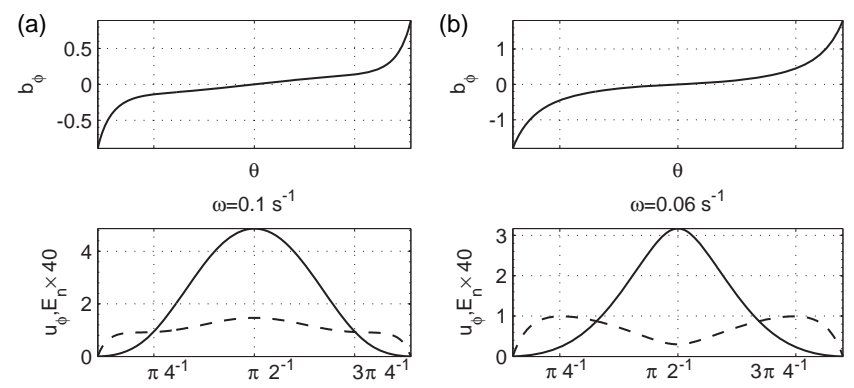

Figure 4. Wave forms of the fundamental mode $(L=6)$ on the (a) noon and (b) midnight meridional plane $(\phi=0$ and $\pi)$, respectively. Dashed line denotes the electric field profile, with a multiplication by 40. All units are arbitrary. The corresponding eigenfrequency is denoted in the middle of each subplot.

seconds at $L=6$. The height (radial distances) of the locations where $\omega_{c}=\omega_{0}$ increase with $L$ values, reaching much greater values in the compressed-dipole case than that in the standard dipole. The corresponding colatitudes, on the other hand, remain close to each other, except for the one near the Equator for $L=6$ on the midnight side of the compresseddipole case.

The choice of $L=6$, which shows the greatest asymmetry between the noon side and midnight side of the compressed dipole, is a representative case to illustrate the spatial wave forms as harmonic solutions to the KG equation. The number of nodes, $n$, contained in the solution of $u_{\phi}$ increases from 0 in the fundamental mode to consecutive positive integral numbers for higher-order harmonics. Figure $4 \mathrm{a}, \mathrm{b}$ show the fundamental modes for the noon and midnight side meridional planes of the compressed dipole. Similar to a standarddipole case (Webb et al., 2012), the $b_{\phi}$ profile contains one node at the Equator, and the oscillating velocity $u_{\phi}$ and electric field $E_{\mathrm{n}}$, normal to the background field (see Fig. 2) are in phase, given the boundary condition $E_{\mathrm{n}}=0$ at both footpoints. The fundamental-mode frequency on the midnight side is a little smaller than the noon side and the corresponding $E_{\mathrm{n}}$ profile has a significant dip (much reduced amplitude) near the Equator. These differences are caused by the different field-line geometry, the critical frequency and the amplitude factor for the two sides as discussed earlier. Such differences persist for higher-order harmonics. Figures 5 and 6 show the wave forms of higher-order harmonics of an increasing number of nodes on the noon and midnight side, respectively. The eigenfrequency increases with an increasing number of nodes. The $u_{\phi}$ and $E_{\mathrm{n}}$ perturbations remain in phase, while the $b_{\phi}$ oscillation is generally out of phase by $\pi / 2$. For the same harmonic mode, the midnight side solution always has a smaller eigenfrequency and a smaller amplitude in $E_{\mathrm{n}}$ around the Equator.
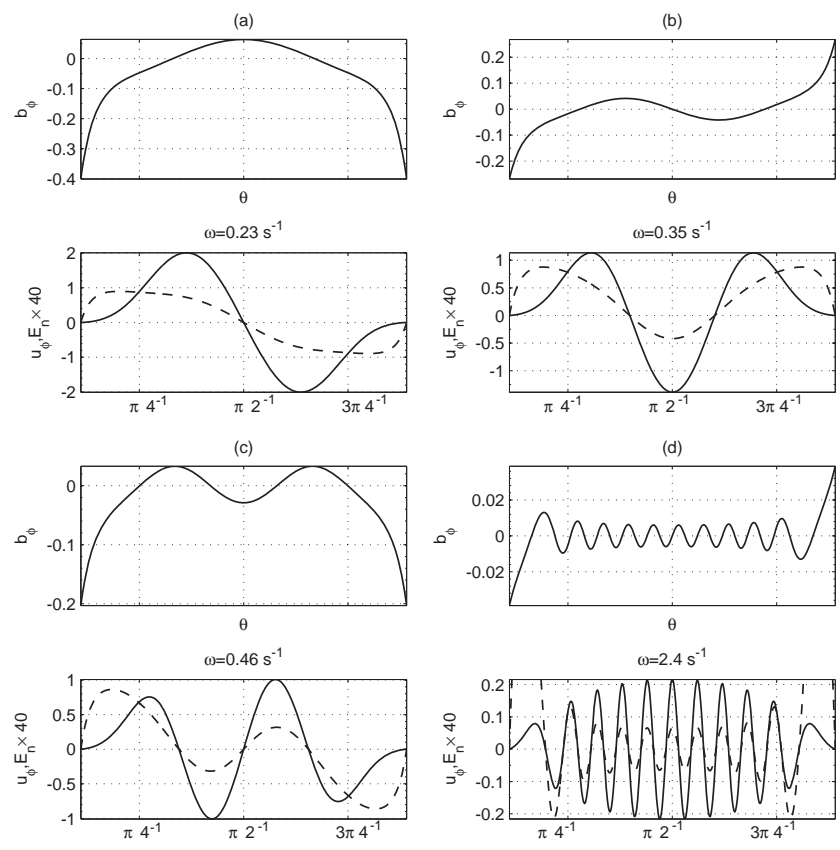

Figure 5. Harmonic wave forms (arbitrary unit) as derived from the solutions of the KG equation on the noon meridional plane $(\phi=0)$ of the compressed-dipole field for (a) $n=1$, (b) $n=2$, (c) $n=3$ and (d) $n>4$. Format is the same as Fig. 4 in each subplot.

\section{Eigenvalue solutions of the poloidal mode}

In the poloidal mode, both the magnetic field and velocity perturbations of the waves are in the meridional plane. The normal components perpendicular to the field line (see Fig. 2) are denoted $b_{n}$ and $u_{n}$, respectively. Therefore, the only oscillating electric field is along the $\hat{\phi}$ direction, $E_{\phi}$ and after multiplied by a scaling factor, $\epsilon_{\phi}=r \sin \theta E_{\phi}$, is governed by (Cummings et al., 1969; Oliver et al., 1993; Lee and Takahashi, 2006)

$\nabla^{2} \epsilon_{\phi}+2 r \sin \theta \nabla \epsilon_{\phi} \cdot \nabla\left(\frac{1}{r \sin \theta}\right)+\frac{\omega^{2}}{V_{A}^{2}} \epsilon_{\phi}=0$,

again assuming a harmonic time variation with angular eigenfrequency, $\omega$. The scaling factor $r \sin \theta$ arises from the curvilinear coordinate system, which is different from a Cartesian geometry. In an equivalent cylindrical coordinate $(R, \phi, Z)(\partial / \partial \phi=0)$, it is written

$\frac{\partial^{2} \epsilon_{\phi}}{\partial R^{2}}-\frac{1}{R} \frac{\partial \epsilon_{\phi}}{\partial R}+\frac{\partial^{2} \epsilon_{\phi}}{\partial Z^{2}}+\frac{\omega^{2}}{V_{A}^{2}} \epsilon_{\phi}=0$.

In a Cartesian geometry, the differential operator in the above equation becomes a single Laplacian and $\epsilon_{\phi} \equiv E_{\phi}$ (e.g., Cummings et al., 1969). Here the Alfvén speed $V_{A}=$ $B / \sqrt{\mu_{0} \rho}$ is again determined by a given background magnetic field and density model, and the equation is solved in 
a 2-D domain such as a meridional plane of the compresseddipole field for $\phi=0$ and $\pi$ only. We are seeking eigenmode solutions subject to boundary condition $\epsilon_{\phi} \equiv 0$ in the present study. Once the electric field perturbation is obtained, the magnetic field perturbations, $b_{r}$ and $b_{\theta}$, lying in the meridional plane, can be derived via Faraday's law using the linear approximations.

Interestingly, the magnetic field perturbation normal to the field line, $b_{n}$, can be derived along each individual field line following the previous approach by the equation below which follows from the linearized Faraday's law:

$$
\frac{\partial b_{n}}{\partial t}=-\frac{B_{\theta}}{r^{2} B \sin \theta} \frac{\mathrm{d} \epsilon_{\phi}}{\mathrm{d} \theta} \text {. }
$$

Note that the total derivative $\mathrm{d} / \mathrm{d} \theta$ here is evaluated along each individual field line and takes the form of Eq. (5). For harmonic oscillations, if we assign a phase lag of $\pi / 2$ to $b_{n}$ relative to $E_{\phi}$ at initial time, the left-hand side of Eq. (23) becomes $\omega \widetilde{b}_{n}$, which allows the derivation of a real-valued amplitude profile of $b_{n}$ based on solutions to Eq. (21). Similarly, the tangential component of the magnetic field perturbation is obtained by

$$
\frac{\partial b_{s}}{\partial t}=\frac{1}{r \sin \theta}\left(\nabla \epsilon_{\phi} \cdot \hat{n}\right)=\frac{1}{r \sin \theta}\left(-\frac{B_{r}}{r B} \frac{\partial \epsilon_{\phi}}{\partial \theta}+\frac{B_{\theta}}{B} \frac{\partial \epsilon_{\phi}}{\partial r}\right) .
$$

In general, the right-hand side of the above equation does not vanish, indicating a compressional fast mode solution. On the other hand, if it does vanish, i.e., $\partial \epsilon_{\phi} / \partial n=0$, a standing Alfvén wave mode should result.

\subsection{Poloidal standing transverse (Alfvén) mode}

This is a special case corresponding to $b_{s} \equiv 0$, i.e., $\partial \epsilon_{\phi} / \partial n=$ 0 from Eq. (24) above. This corresponds to a transverse, Alfvén mode of poloidal polarization of the magnetic field perturbation that propagates along individual field lines. Therefore, we can apply exactly the same approach of Sect. 2. The electric field perturbation $\epsilon_{\phi}$ still satisfies Eq. (21). However, when applying the condition $b_{s}=0$ and projecting the partial differential equation (PDE) along an individual field line defined by a relation between $r$ and $\theta$, a wave equation of the form similar to Eq. (6) is obtained:

$$
\frac{\partial^{2} \epsilon_{\phi}}{\partial t^{2}}=\frac{V^{2}}{r^{2}}\left[\frac{\mathrm{d}^{2} \epsilon_{\phi}}{\mathrm{d} \theta^{2}}-\frac{\mathrm{d}}{\mathrm{d} \theta} \ln \left(\frac{B^{2}}{B_{\theta}^{2}} g(\theta) \sin \theta\right) \frac{\mathrm{d} \epsilon_{\phi}}{\mathrm{d} \theta}\right] .
$$

Here the wave speed parameter $V^{2} \equiv B_{\theta}^{2} /\left(\mu_{0} \rho\right)$ remains the same as before, and the function $g(\theta)$ is determined from a given background magnetic field model along an individual field line $r(\theta)$ by

$$
\frac{\mathrm{d}}{\mathrm{d} \theta} \ln g(\theta)=\frac{B_{r}^{2}}{B_{\theta}^{2}} \frac{\partial}{\partial r}\left(\frac{r B_{\theta}}{B_{r}}\right) .
$$

For example, for a standard-dipole field, the function $g(\theta)=$ $\sin ^{2} \theta$ is obtained.
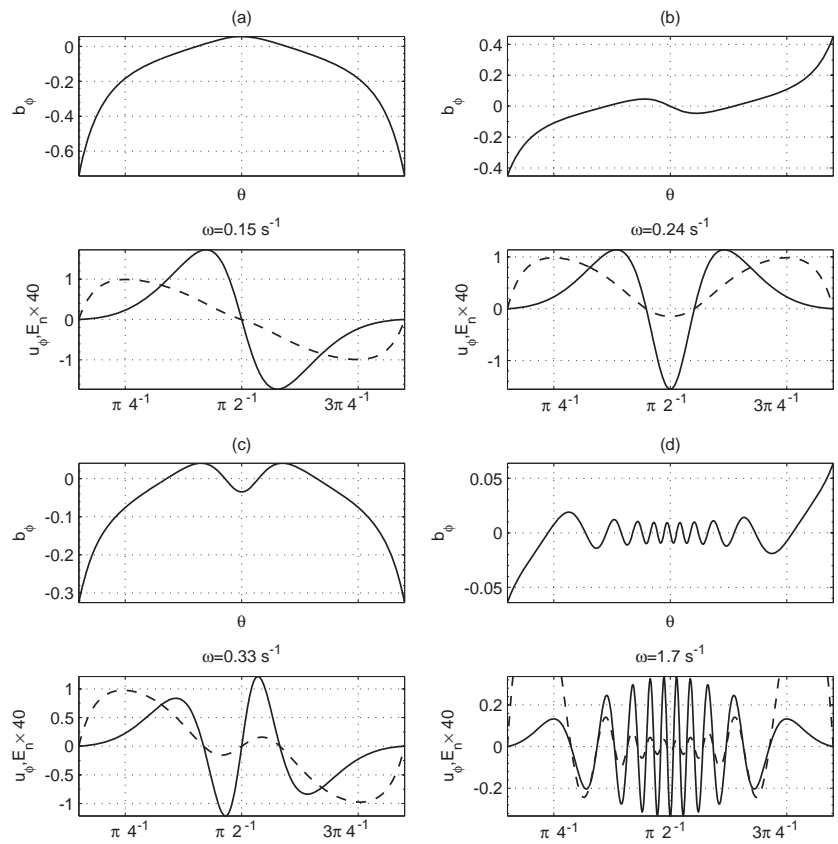

Figure 6. Harmonic wave forms on the midnight meridional plane $(\phi=\pi)$ of the compressed-dipole field. Format is the same as Fig. 5.

Therefore, the wave Eq. (25) can also be cast as a KG form and solved for eigenvalue solutions subject to the boundary condition $\epsilon_{\phi}=0$ at the footpoints of an individual field line. In turn the magnetic field perturbation can be derived from Eq. (23). Below, we list the essential parameters for this mode conforming to the general descriptions in Sect. 2.1:

$$
\begin{aligned}
\frac{1}{L_{\epsilon}} & =\frac{\mathrm{d}}{\mathrm{d} \theta} \ln \left(\frac{B^{2}}{B_{\theta}^{2}} g(\theta) \sin \theta\right), \\
\frac{1}{M_{\epsilon}} & =0
\end{aligned}
$$

and the amplitude factor

$$
f(\theta)=\frac{B}{B_{\theta}} \sqrt{g(\theta) \sin \theta}
$$

For the dipole field, the following explicit formulas are obtained

$$
f(\theta)=\sqrt{\sin \theta\left(1+3 \cos ^{2} \theta\right)},
$$

$$
\frac{1}{L_{\epsilon}}=\cot \theta-\frac{3 \sin 2 \theta}{1+3 \cos ^{2} \theta} .
$$

Thus, the critical frequency $\omega_{c}$ can be written based on Eq. (9). Figure 7 shows, in the same format as before, the profiles of $\omega_{c}$ and $f$ for selected $L$ shells of the dipole field. 

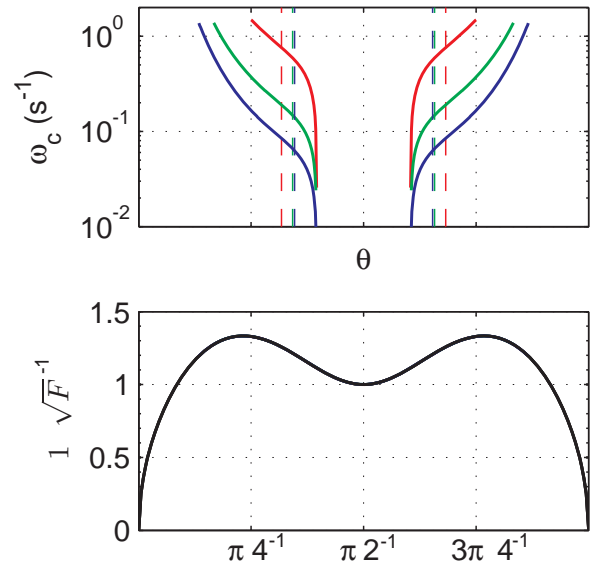

Figure 7. The parameters $\omega_{c}$ and $f(\theta)$ for the poloidal standing Alfvén waves of the dipole field. Format is the same as Fig. 1.

Similarly, the critical frequency decreases from the poles toward the Equator where $\omega_{c}^{2}$ becomes negative. The eigenfrequency of the fundamental mode generally intersects the critical frequency at mid- to low latitudes. The solution of the KG equation would also be a combination of a propagation type near the Equator and a decaying type near the two ends. The amplitude factor $f(\theta)$, on the other hand, shows much less variation in magnitude and does not depend on $L$. Figure 8 shows the fundamental-mode solutions for $L=5$, typical of a standing wave with zero nodes in electric field perturbation. The amplitude of $E_{\phi}$ dips slightly around the Equator. The eigenfrequency is $0.092 \mathrm{~s}^{-1}$, which corresponds to a period of $68 \mathrm{~s}$. It compares well with observations to be discussed below. Table 4 lists the corresponding parameters for the selected $L$ shells in the same format as Tables $1-3$. The periods are in the same range as those of the toroidal mode.

For a compressed-dipole field, because the relation below $r$ and $\theta$ along a field line is implicit, the relevant quantities have to be evaluated numerically, especially the function $g(\theta)$ according to Eq. (26). In what follows, we provide solutions to the $\mathrm{KG}$ equation for the poloidal transverse mode in a compressed-dipole field with the magnetic field components given by Eqs. (14) to (16).

In this case, Eq. (26) becomes

$$
\frac{\mathrm{d}}{\mathrm{d} \theta} \ln g(\theta)=\frac{2+10 D r^{3}-D^{2} r^{6}}{\left(1+D r^{3}\right)^{2}} \cot \theta=h(\theta),
$$

where the radial distance $r$ implicitly depends on $\theta$ as indicated by Eq. (18) along an individual field line. Therefore, the function $g(\theta)$ has to be obtained by numerical integration of $h(\theta)$. Subsequently the coefficient functions $1 / L_{\epsilon}$ and its derivative with respect to $\theta$ appearing in the $\mathrm{KG}$ equation can be efficiently and accurately estimated by numerical differentiations.

The variations in the critical frequency $\omega_{c}$ along individual field lines of $L=2,4,6$ for both the noon and midnight
Table 4. List of parameters for $L=2,4,6$ of a dipole field for poloidal standing Alfvén waves.

\begin{tabular}{ccccc}
\hline$L$ & $\omega_{0}, \mathrm{~s}^{-1}$ & $T_{0}, \mathrm{~s}$ & $\theta_{0},{ }^{\circ}$ & $r_{0}\left(R_{\mathrm{E}}\right)$ \\
\hline 2 & 0.76 & 8.2 & 57 & 1.41 \\
4 & 0.15 & 42 & 62 & 3.09 \\
6 & 0.062 & 101 & 62 & 4.71 \\
\hline
\end{tabular}

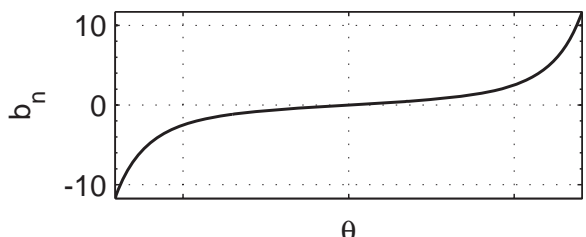

$\theta$

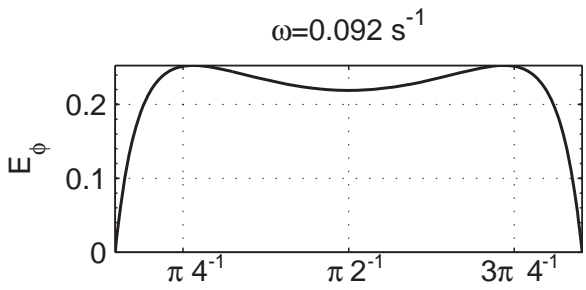

Figure 8. Wave forms (arbitrary unit) of the fundamental mode ( $L=5$ ) for the poloidal standing Alfvén mode of the dipole field. Format is the same as Fig. 4.

sides are shown in Fig. 9, together with the amplitude factor $f(\theta)$. Compared with the corresponding variations of the dipole case in Fig. 7, the critical frequency again exhibits gaps where $\omega_{c}^{2}<0$, but the profiles of the amplitude factor become dependent on $L$, for both sides. Then the KG equation is solved for $L=5\left(M_{\epsilon}=0\right)$ and the solutions corresponding to the fundamental mode are given in Fig. 10. They exhibit very similar behavior to the solution of the dipole field given in Fig. 8 except that the corresponding eigenfrequencies are different such that the one for the dipole field is in-between the ones for the compressed-dipole cases. The corresponding periods of the compressed-dipole cases are $52 \mathrm{~s}$ and $93 \mathrm{~s}$, respectively, for the noon and midnight side. It is worth noting that for these poloidal modes of different background field configurations, gaps always exist in the critical frequencies where $\omega_{c}^{2}<0$. Therefore, the propagatingtype solutions corresponding to $\omega^{2}>\omega_{c}^{2}$ always exist in this mode. In other words, there is no lower bound in these cases on the wave frequency.

\subsection{A real-case study of poloidal standing Alfvén mode}

In this subsection, we demonstrate the validity of our approach by comparing our analysis result with a recent direct spacecraft observation of poloidal standing Alfvén waves by Dai et al. (2013). Since these observations occurred near $\sim 8.5$ in magnetic local time (MLT) of the Earth's magne- 


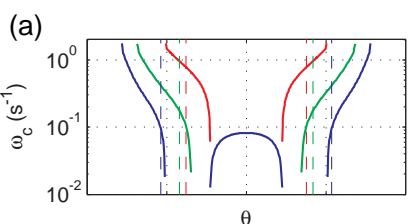

(b)
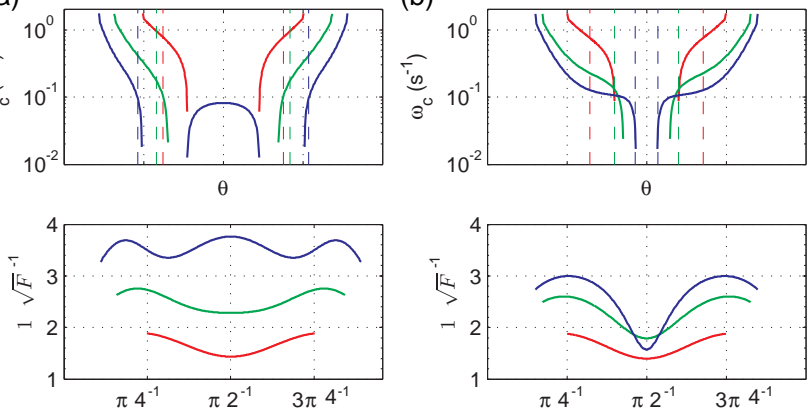

Figure 9. The parameter $\omega_{c}$ and the adiabatic growth and decay factor as a function of $\theta$ for various $L$ values of the poloidal transverse mode for the compressed-dipole field with (a) $\phi=0$ and (b) $\phi=\pi$, respectively. Format is the same as Fig. 1. The broken part of some curves in top panels corresponds to $\omega_{c}^{2}<0$.

tosphere, we present the analysis results of the dipole and the compressed-dipole field, which offer different background field geometries. Figure 11 shows our results of suitable physical units for $L=5$ with the same set of parameters as Dai et al. (2013), $\rho_{e}=6.4 \mathrm{amu} \mathrm{cm}^{-3}$ and the power index 1.0 of the density variation, to facilitate a direct comparison with their results (Fig. 3 therein). Dai et al. (2013) used a theoretical model of Cummings et al. (1969) and realistic ionosphere boundary conditions of finite conductivity at the footpoints of the field line. Therefore, their solutions of $E_{\phi}$ and $b_{n}$ profiles are of non-zero values at the ends and are asymmetric about the Equator, whereas ours are symmetric and $E_{\phi}$ vanishes at the two ends. Nonetheless the spatial profiles over the mid- to low latitudes still compare very well. In both columns, the magnitudes of both perturbations show a slight decrease toward the Equator in $E_{\phi}$ and a rapid increase toward the ends in $b_{n}$. In particular, the wave periods of the two cases are $45 \mathrm{~s}$ and $82 \mathrm{~s}$, while the observed value is $84 \mathrm{~s}$, closer to the period of the midnight side. The ratios of $\left|b_{n} / E_{\phi}\right|$ at the spacecraft location $\left(\sim 17^{\circ}\right.$ south in latitude $)$ are 0.15 and $0.30 \mathrm{nT} /(\mathrm{mV} / \mathrm{m})$ from Fig. 11a and b, respectively, while the observed value is $0.3 \mathrm{nT} /(\mathrm{mV} / \mathrm{m})$, identical to the latter of the ratios. Our result is also consistent with the observation in that $E_{\phi}$ leads the phase of $b_{n}$ by $\pi / 2$ as discussed earlier. The corresponding wave period and amplitude ratio of the fundamental poloidal mode for the standarddipole field by using the above set of parameters are 60s and $0.21 \mathrm{nT} /(\mathrm{mV} / \mathrm{m})$, given in Fig. 12, respectively, values which are somewhere in between those values presented for the two sides of the compressed dipole. The estimates of these quantities in Dai et al. (2013) by a different theoretical model are $62 \mathrm{~s}$ and $0.25 \mathrm{nT} /(\mathrm{mV} / \mathrm{m})$, respectively. Thus, this particular ULF wave observation is consistent with our model results.
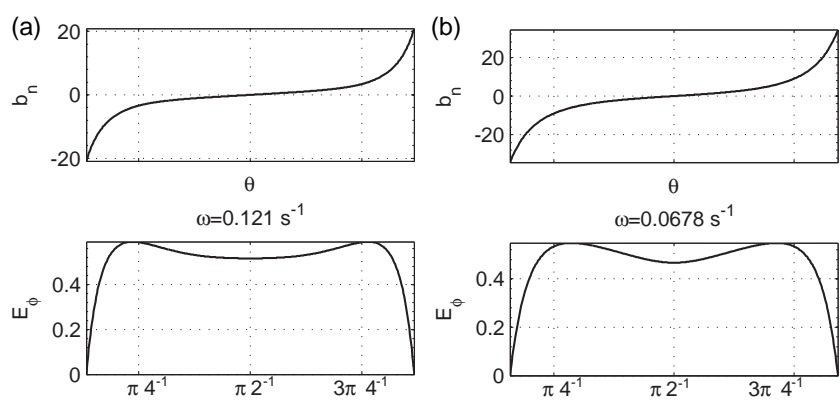

Figure 10. Wave forms of the poloidal fundamental mode $(L=5)$ on the (a) $\operatorname{noon}(\phi=0)$ and (b) midnight $(\phi=\pi)$ meridional plane, respectively. Format is the same as Fig. 8.
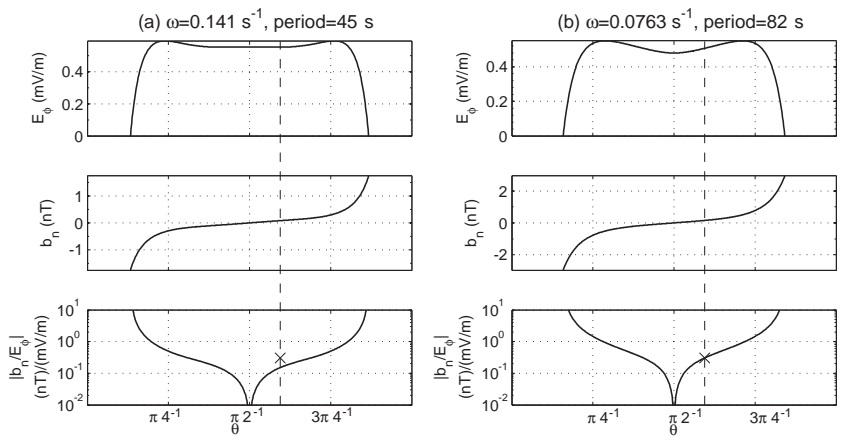

Figure 11. The solutions of $E_{\phi}$ (in $\mathrm{mV} / \mathrm{m}$ ), $b_{n}$ (in $\mathrm{nT}$ ) and the ratio inbetween (from top to bottom panels) for the compressed dipole on the (a) noon side and (b) midnight side, to be compared with the spacecraft observations of Dai et al. (2013). The vertical dashed line denotes the spacecraft location and the cross marks the measured ratio $(\sim 0.3 \mathrm{nT} /(\mathrm{mV} / \mathrm{m}))$ during the time period of measurements.

\subsection{Poloidal compressional mode}

In the case that $b_{s} \neq 0$, Eq. (22) has to be solved in a 2-D domain as an eigenvalue problem subject to the boundary condition $E_{\phi}=0$ on all sides. We have solved the equation and obtained the corresponding eigenmode solutions of a discrete set of increasing eigenfrequencies by utilizing the software package PDE2D. ${ }^{1}$ The solutions are also crosschecked with the Matlab PDE toolbox and identical results are obtained. The computational domain is chosen as $r \in$ $\left[1, L_{p}\right] R_{\mathrm{E}}$ and $\theta \in\left[\theta_{p}, \pi-\theta_{p}\right]$, where $\theta_{p}=\arcsin \sqrt{1 / L_{p}}$. We choose $L_{p}=7$ in order to avoid the singular point in the compressed-dipole field model as well as the singularity along the poles $(X=0)$. We apply the dipole field and the compressed-dipole field models and the same density distribution as before for the background field and plasma conditions. Three sets of eigenfrequencies of ascending order of magnitude (mode) are obtained for both the noon and midnight side meridional planes of the compressed-dipole field and the standard-dipole field. The first 100 eigenfrequencies

\footnotetext{
${ }^{1}$ http://www.pde2d.com/
} 


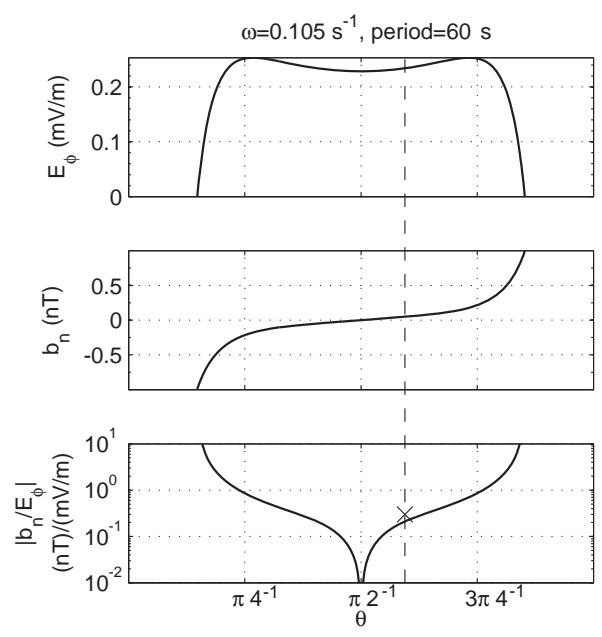

Figure 12. The solutions of $E_{\phi}$ (in $\mathrm{mV} / \mathrm{m}$ ), $b_{n}$ (in $\mathrm{nT}$ ) and the ratio inbetween (from top to bottom panels) for the standard dipole of the same set of parameters as Dai et al. (2013). Format is the same as Fig. 11.

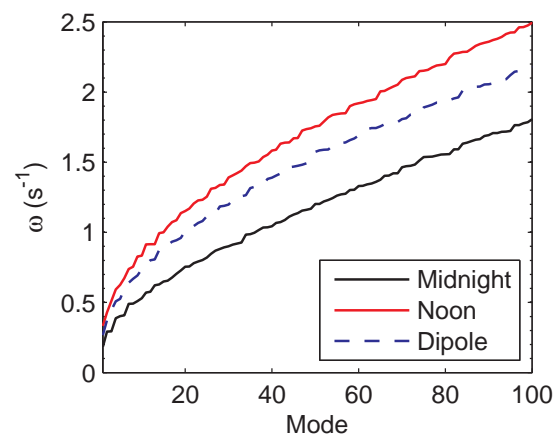

Figure 13. The first 100 eigenfrequencies for the noon side and midnight side of the compressed dipole and a standard-dipole field.

are shown in Fig. 13. They generally exhibit a rapid rise at the lowest numbers of mode; then the trend of increase seems to become more gradual and eventually linear. At one specific mode, the eigenfrequency of the noon side is always greater than that of the midnight side, while the value of the dipole case is always inbetween, albeit slightly closer to the value of the noon side. The corresponding eigenmode solutions (not shown) generally display a regular pattern of nodal structures of progressively increasing number of nodes with increasing eigenfrequencies. Our preliminary numerical experiments indicate that the background magnetic field greatly affects the eigenvalue solutions.

\section{Conclusions and discussion}

In conclusion, we have examined, in a fairly comprehensive manner, the decoupled toroidal and poloidal-mode hydromagnetic waves in cold plasmas with applications to the Earth's inner magnetosphere (ULF waves), represented by a compressed-dipole field model in addition to the standard-dipole field. Under certain assumptions, the decoupled wave equations are recast as the Klein-Gordon (KG) form along individual magnetic field lines, especially for both the toroidal and poloidal transverse Alfvén waves. Such a KG equation describes the effect of background medium on wave propagation (Morse and Feshbach, 1953), as embodied in the term involving the critical frequency, $\omega_{c}$. We obtain the spatial profiles of the characteristic parameters in the KG formulations including the critical frequency $\omega_{c}$ and the amplitude factor $f$, both as functions of the colatitude, $\theta$. The former generally exhibits a behavior of minimum values below $0.1 \mathrm{~Hz}$ (sometimes $0.01 \mathrm{~Hz}$ ) near the Equator and increasing values towards the footpoints, exceeding $1 \mathrm{~Hz}$. This leads to a spatially composite solution of a propagating type where the eigenfrequency $\omega>\omega_{c}$ usually occurs near the Equator and a decaying type where $\omega<\omega_{c}$ occurs toward the footpoints. The latter modulates the amplitude of the wave forms spatially. We obtain the sets of eigenvalue solutions of increasing eigenfrequencies and the number of nodes in the wave forms for different background magnetic field geometries. The corresponding wave periods are on the order of $\sim 1$ to $\sim 100 \mathrm{~s}$ and compare well quantitatively with prior studies and observations. In particular, we present a case study of a fundamental poloidal Alfvén wave via our approach and compare our results with a direct spacecraft observation by Dai et al. (2013). The observed wave period $\sim 84 \mathrm{~s}$ and the amplitude ratio $\sim 0.3 \mathrm{nT} /(\mathrm{mV} / \mathrm{m})$ agree with our ranges of estimates $-45-82 \mathrm{~s}$ and $0.15-0.3 \mathrm{nT} /(\mathrm{mV} / \mathrm{m})$, respectively. Thus, we provide a relatively simple yet reliable means of analyzing ULF wave observations in the magnetosphere.

The main intellectual merits of this work lie in the aspect of the unique approach via the KG equations for both the toroidal and poloidal transverse Alfvén waves for a given background magnetic field geometry. The existence of the critical frequency indicates the importance of the background magnetic field topology. For some instances, a propagating wave mode can only exist above a certain frequency, i.e., the minimum $\omega_{c}$. In particular, the case study of a direct comparison with spacecraft measurements yields consistent results. However, discrepancies probably occur due to the relatively simple and idealized assumptions about the axisymmetric geometry, the background and boundary conditions. A brief discussion of wave energy in terms of wave mixing was given in Webb et al. (2012). Since we only deal with standing waves in the present work, no net energy flow results after time average, irrespective of the critical frequency. The study of ULF waves is worth pursuing beyond the limitations of the present approach, especially in conjunction with state-of-theart spacecraft observations, such as those returned from the Van Allen Probes. More realistic study of energy transport considering nonideal ionospheric boundary conditions will be pursued in future work. Furthermore, it is also desirable 
to extend the applications to the solar coronal loop oscillations as we did in Hu et al. (2012).

Acknowledgements. We dedicate this paper to the late James F. McKenzie, who initiated this work during his visit and was partially supported by the Pei-Ling Chan Chair of Physics of the University of Alabama in Huntsville and the NRF of South Africa. His infectious enthusiasm for the subject motivated us to complete this work in his honor. He will be missed as a colleague, a mentor and a friend. The other authors acknowledge partial support of NSF grant AGS-1062050 and NASA grant NNX12AH50G. J. Zheng especially acknowledges partial support of a graduate research assistantship through the NASA grant. We are also grateful to G. Sewell for his help with the PDE2D software package. Q. Hu acknowledges useful discussions with C.-S. Wu, Bo Li, Yao Chen and Lidong Xia and is grateful for their hospitality during his visit to Shandong University, Weihai, China. We also thank the reviewers for their constructive comments.

The topical editor, G. Balasis, thanks the three anonymous referees for help in evaluating this paper.

\section{References}

Chen, L. and Cowley, S. C.: On field line resonances of hydromagnetic Alfven waves in dipole magnetic field, Geophys. Res. Lett., 16, 895-897, doi:10.1029/GL016i008p00895, 1989.

Claudepierre, S. G., Hudson, M. K., Lotko, W., Lyon, J. G., and Denton, R. E.: Solar wind driving of magnetospheric ULF waves: Field line resonances driven by dynamic pressure fluctuations, J. Geophys. Res.-Space, 115, A11202, doi:10.1029/2010JA015399, 2010.

Cummings, W. D., O’Sullivan, R. J., and Coleman, Jr., P. J.: Standing Alfvén waves in the magnetosphere, J. Geophys. Res., 74, 778, doi:10.1029/JA074i003p00778, 1969.

Dai, L., Takahashi, K., Wygant, J. R., Chen, L., Bonnell, J., Cattell, C. A., Thaller, S., Kletzing, C., Smith, C. W., MacDowall, R. J., Baker, D. N., Blake, J. B., Fennell, J., Claudepierre, S., Funsten, H. O., Reeves, G. D., and Spence, H. E.: Excitation of poloidal standing Alfvén waves through drift resonance wave-particle interaction, Geophys. Res. Lett., 40, 4127-4132, doi:10.1002/grl.50800, 2013.

Dai, L., Takahashi, K., Lysak, R., Wang, C., Wygant, J. R., Kletzing, C., Bonnell, J., Cattell, C. A., Smith, C. W., MacDowall, R. J., Thaller, S., Breneman, A., Tang, X., Tao, X., and Chen, L.: Storm time occurrence and spatial distribution of Pc4 poloidal ULF waves in the inner magnetosphere: A Van Allen Probes statistical study, J. Geophys. Res.-Space, 120, 47484762, doi:10.1002/2015JA021134, 2015.

Degeling, A. W., Rankin, R., Kabin, K., Rae, I. J., and Fenrich, F. R.: Modeling ULF waves in a compressed dipole magnetic field, J. Geophys. Res.-Space, 115, A10212, doi:10.1029/2010JA015410, 2010.

Dimitrakoudis, S., Mann, I. R., Balasis, G., Papadimitriou, C., Anastasiadis, A., and Daglis, I. A.: Accurately specifying stormtime ULF wave radial diffusion in the radiation belts, Geophys. Res. Lett., 42, 5711-5718, doi:10.1002/2015GL064707, 2015.
Dungey, J. W.: Electrodynamics of the Outer Atmosphere, in: Physics of the Ionosphere, London, The Physical Society, 229 pp., 1955.

Dungey, J. W.: Hydromagnetic waves and the ionosphere, in: The Ionosphere, London: The Institute of Physics and the Physical Society, edited by: Stickland, A. C., 230 pp., 1963.

Elkington, S. R.: A Review of ULF Interactions With Radiation Belt Electrons, in: Magnetospheric ULF Waves: Synthesis and New Directions, edited by: Takahashi, K., Chi, P. J., Denton, R. E., and Lysak, R. L., 169 , Washington DC American Geophysical Union Geophysical Monograph Series, 177 pp., 2006.

Elkington, S. R., Hudson, M. K., and Chan, A. A.: Acceleration of relativistic electrons via drift-resonant interaction with toroidalmode Pc-5 ULF oscillations, Geophys. Res. Lett., 26, 32733276, doi:10.1029/1999GL003659, 1999.

Elkington, S. R., Hudson, M. K., and Chan, A. A.: Resonant acceleration and diffusion of outer zone electrons in an asymmetric geomagnetic field, J. Geophys. Res.-Space, 108, 1116, doi:10.1029/2001JA009202, 2003.

Fraser, B. J.: ULF Waves-A Historical Note, in: Magnetospheric ULF Waves: Synthesis and New Directions, edited by: Takahashi, K., Chi, P. J., Denton, R. E., and Lysak, R. L., 169, Washington DC American Geophysical Union Geophysical Monograph Series, p. 5, 2006.

Hu, Q., McKenzie, J. F., and Webb, G. M.: Klein-Gordon equations for horizontal transverse oscillations in two-dimensional coronal loops, Astron. Astrophys., 541, A53, doi:10.1051/00046361/201117421, 2012.

Kabin, K., Rankin, R., Mann, I. R., Degeling, A. W., and Marchand, R.: Polarization properties of standing shear Alfvén waves in non-axisymmetric background magnetic fields, Ann. Geophys., 25, 815-822, doi:10.5194/angeo-25-815-2007, 2007.

Kivelson, M. G.: ULF waves from the ionosphere to the outer planets, in: Magnetospheric ULF Waves: Synthesis and New Directions, edited by: Takahashi, K., Chi, P. J., Denton, R. E., and Lysak, R. L., 169, Washington DC American Geophysical Union Geophysical Monograph Series, 11-30, doi:10.1029/169GM04, 2006.

Lee, D.-H. and Takahashi, K.: MHD Eigenmodes in the Inner Magnetosphere, in: Magnetospheric ULF Waves: Synthesis and New Directions, edited by: Takahashi, K., Chi, P. J., Denton, R. E., and Lysak, R. L., 169, Washington DC American Geophysical Union Geophysical Monograph Series, p. 73, 2006.

Liu, W., Sarris, T. E., Li, X., Zong, Q.-G., Ergun, R., Angelopoulos, V., and Glassmeier, K. H.: Spatial structure and temporal evolution of a dayside poloidal ULF wave event, Geophys. Res. Lett., 38, L19104, doi:10.1029/2011GL049476, 2011.

Liu, W., Cao, J. B., Li, X., Sarris, T. E., Zong, Q.-G., Hartinger, M., Takahashi, K., Zhang, H., Shi, Q. Q., and Angelopoulos, V.: Poloidal ULF wave observed in the plasmasphere boundary layer, J. Geophys. Res.-Space, 118, 4298-4307, doi:10.1002/jgra.50427, 2013.

McKenzie, J. F. and Hu, Q.: Klein-Gordon equations for toroidal hydromagnetic waves in an axi-symmetric field, Ann. Geophys., 28, 737-742, doi:10.5194/angeo-28-737-2010, 2010.

Morse, P. M. and Feshbach, H.: Methods of theoretical physics, vol. 1, chap. 7, 854-857, McGraw-Hill Book Company, INC., Tokyo, Japan, 1 edn., 1953. 
Oliver, R., Ballester, J. L., Hood, A. W., and Priest, E. R.: Magnetohydrodynamic waves in a potential coronal arcade, Astron. Astrophys., 273, 647-658, 1993.

Singer, H. J., Southwood, D. J., Walker, R. J., and Kivelson, M. G.: Alfven wave resonances in a realistic magnetospheric magnetic field geometry, J. Geophys. Res., 86, 4589-4596, doi:10.1029/JA086iA06p04589, 1981.

Southwood, D. J. and Hughes, W. J.: Theory of hydromagnetic waves in the magnetosphere, Space Sci. Rev., 35, 301-366, doi:10.1007/BF00169231, 1983.

Takahashi, K., Denton, R. E., and Gallagher, D.: Toroidal wave frequency at $\mathrm{L}=6-10$ : Active Magnetospheric Particle Tracer Explorers/CCE observations and comparison with theoretical model, J. Geophys. Res.-Space, 107, 1020, doi:10.1029/2001JA000197, 2002.

Takahashi, K., Hartinger, M. D., Angelopoulos, V., Glassmeier, K.H., and Singer, H. J.: Multispacecraft observations of fundamental poloidal waves without ground magnetic signatures, J. Geophys. Res.-Space, 118, 4319-4334, doi:10.1002/jgra.50405, 2013.
Tamao, T.: Transmission and coupling resonance of hydromagnetic disturbances in the non-uniform Earth's magnetosphere, Sci. Rep. Tohoku Univ., Ser. 5, Geophys., 17, 43-72, 1965.

Ukhorskiy, A. Y., Takahashi, K., Anderson, B. J., and Korth, H.: Impact of toroidal ULF waves on the outer radiation belt electrons, J. Geophys Res.-Space, 110, A10202, doi:10.1029/2005JA011017, 2005.

Volwerk, M.: Multi-Satellite Observations of ULF Waves, in: Magnetospheric ULF Waves: Synthesis and New Directions, edited by Takahashi, K., Chi, P. J., Denton, R. E., and Lysak, R. L., vol. 169, Washington DC American Geophysical Union Geophysical Monograph Series, p. 109, 2006.

Webb, G. M., McKenzie, J. F., Hu, Q., and Zank, G. P.: Toroidal hydromagnetic waves in an axi-symmetric magnetic field, J. Geophys. Res.-Space, 117, A05229, doi:10.1029/2012JA017561, 2012. 